\title{
Frequenza delle complicanze della biopsia renale con tecnica chirurgica, transcutanea ecoguidata: studio retrospettivo
}

\author{
G.P. Lupi, S. Bisegna, L. Cornalba, C. Grassi \\ Servizio di Nefrologia e Dialisi Ospedale Predabissi Melegnano
}

a biopsia renale rappresenta un'indagine insostituibile nella valutazione diagnostica, prognostica e terapeutica dei pazienti nefropatici.

Dal 1923, anno in cui fu eseguita la prima biopsia renale con tecnica chirurgica (1), la metodica si è evoluta con l'introduzione nel 1934 della biopsia renale percutanea (2), che diventa routinaria negli anni '50 (3-5). Nel 1954 Kark and Muehrcke modificano la procedura effettuando la biopsia con paziente in posizione prona anzicché seduta, utilizzando un ago fine per localizzare il rene prima di introdurre l'ago per biopsia (6). Dal 1961, dopo il Simposio Ciba sulla biopsia renale tenuto a Boston, viene introdotta definitivamente la metodica bioptica transcutanea (7). L'avvento della ultrasonografia renale ha reso più facile l'individuazione del rene, e la disponibilità di adattatori per aghi da inserire sulle sonde ha ulteriormente semplificato la tecnica stessa (8-12).

La manovra bioptica non è comunque esente da complicanze. Esse annoverano la macroematuria, l'ematoma perirenale, la fistola artero-venosa e più raramente la puntura accidentale di fegato, milza, pancreas ed intestino, il pneumotorace e le infezioni delle vie urinarie, soprattutto in pazienti affetti da pielonefrite (13-18). Il rischio di nefrectomia e di morte è estremamente basso. In una recente casistica riguardante 14.500 biopsie pubblicate in letteratura, esso è risultato essere rispettivamente dello $0.3 \%$ e $0.1 \%$ (19). Scopo del presente lavoro è l'analisi della prevalenza delle complicanze della biopsia renale ed il rilievo di loro eventuali differenze nelle tre tecniche bioptiche utilizzate dal nostro servizio: chirurgica (BRC), transcutanea (BRT) e transcutanea ecoguidata (BRTE).

\section{Materiali e metodi}

Per valutare la prevalenza delle complicanze della biopsia renale eseguita su pazienti nefropatici afferiti al nostro centro a partire dal 1980 , sono state analizzate retrospettivamente 131 biopsie (chirurgica 24 , transcutanea 26 , transcutanea ecoguidata 81 ), eseguite a 126 pazienti (M 80 - F 46) di età compresa tra 9 e 84 anni. La distribuzione delle nefropatie causali era la seguente (Fig.1): IgA 31, M. Change 10, LES 10, Membranosa 13, Glomerulosclerosi focale 10, Glomerulonefriti non classificate 10 , Crioglobulinemia 5, Membrano Proliferativa I-II-III 5, Good-Pasture 3, Glomerulonefrite extracapillare 6 , Glomerulonefrite post-infettiva 2, Amiloidosi $\mathrm{I}^{\circ}$ 2, Diabete ID 1, SEU 2, S. Henoch 1, S. Alport 1, Amiloidosi $\mathrm{II}^{\circ} 1$, Wegener 2, Altre 3, materiale inadeguato 5 .

Tutti i soggetti erano stati sottoposti ad osservazione clinica, controllo della

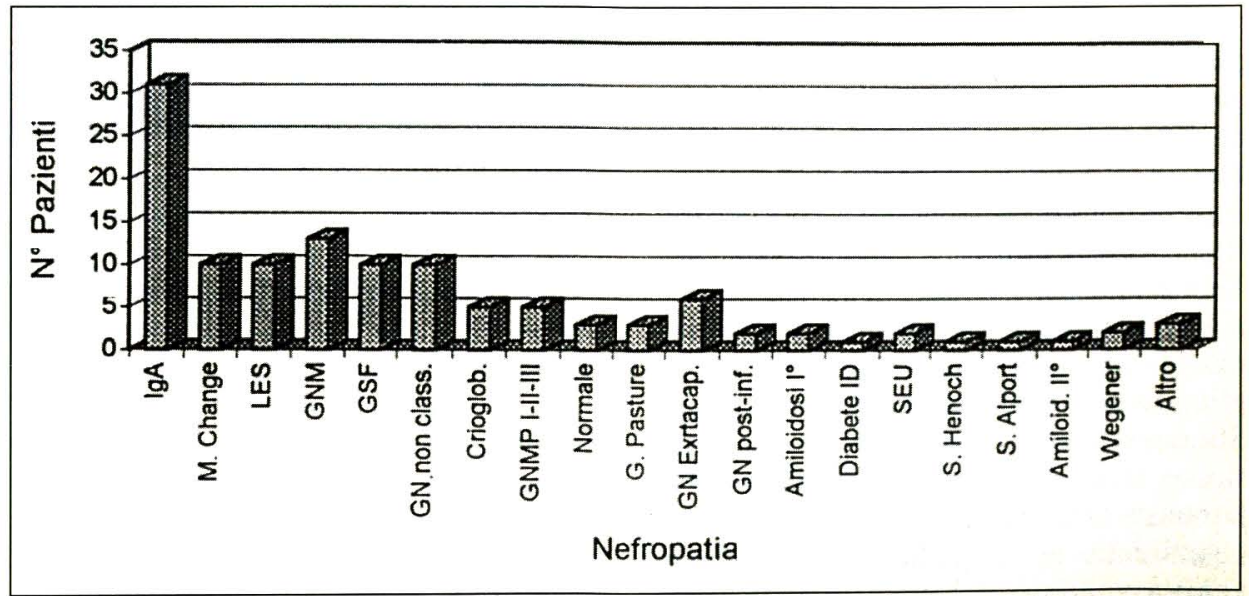

Fig. 1 - Distribuzione per nefropatia 


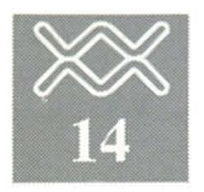

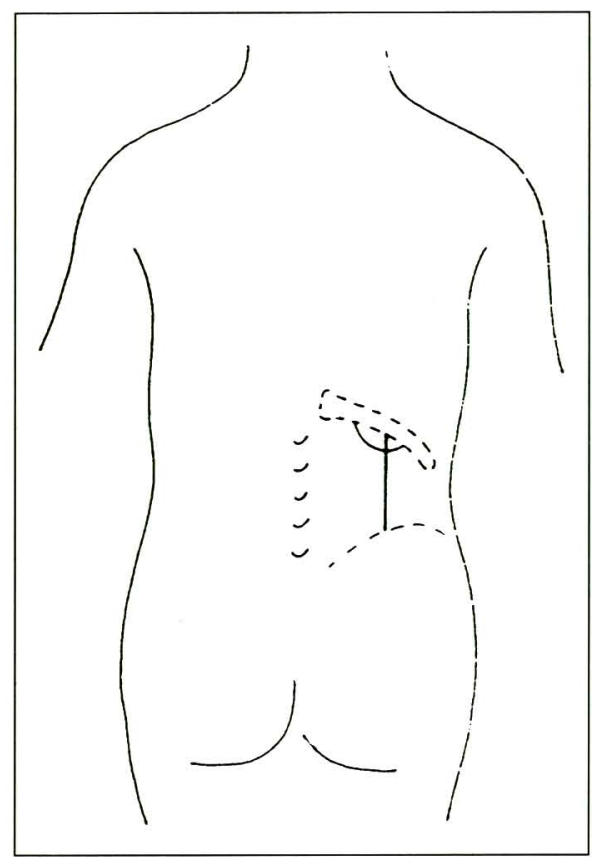

Fig. 2 - Incisione cutanea verticale posteriore secondo Simon.

pressione arteriosa $(<140 / 90)$ e della crasi ematica (emocromo, AP-PTT,e tempo di emorragia sec Ivy) prima della manovra bioptica. I pazienti venivano tenuti in osservazione nelle 36 ore successive la biopsia, durante le quali venivano valutate la quantità della diuresi e la presenza o meno di macroematuria; venivano inoltre effettuati un esame emocromocitometrico ed una ecografia di controllo prima della dimissione, per individuare l'eventuale comparsa di ematoma perirenale. La tecnica chirurgica veniva eseguita previa lombotomia posteriore secondo Simon (verticale) (Fig.2) o la classica incisione sottocostale obliqua. Per la biopsia transcutanea veniva utilizzato un ago Tru-Cut 16 Gauge. La biopsia transcutanea ecoguidata veniva eseguita con apparecchio ecografico Toshiba Sonolayer SSA-250 K con sonda Convex $3.5 \mathrm{Mhz}$, adattatore per biopsia Toshiba ed ago semiautomatico TemnoBauer 15-16 gauce con punta ecogena. Tale tecnica consisteva nella localizzazione ecografica del polo inferiore del rene sinistro e nella valutazione del miglior angolo di penetrazione dell'ago. Successivamente veniva riportato lo stesso angolo sull'adattatore ed iniziata la manovra bioptica. Il tragitto dell'ago, posizionato nella guida dell'adattatore, veniva valutato sul monitor dell'ecografo (Figg. 3, 4).

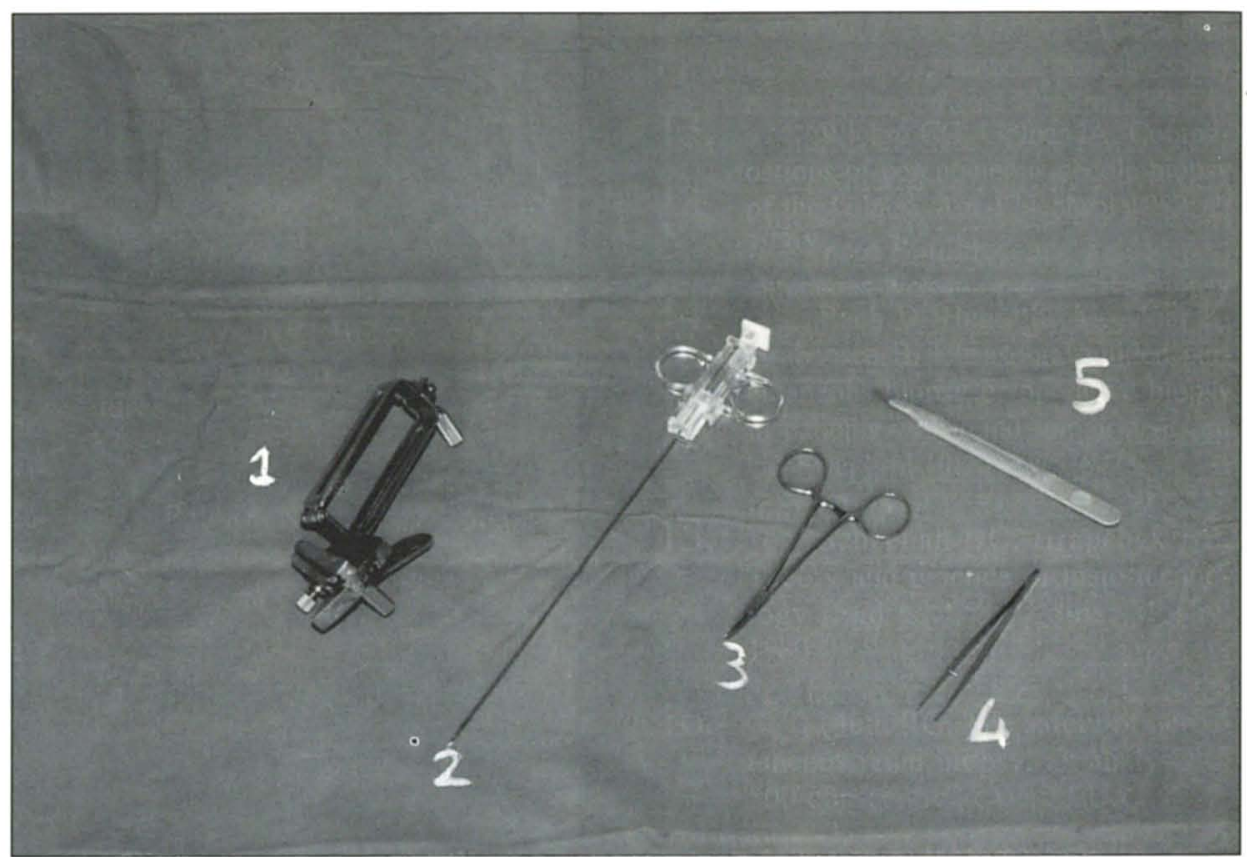

Fig. 3 - Materiale utilizzato per la biopsia renale: 1 Adattatore Toshiba per ago, 2 Ago Temno-Bauer semiautomatico centimetrato con punta ecogena, 3 Klemmer curvo, 4 Pinza per raccolta frustolo bioptico, 5 Bisturi.

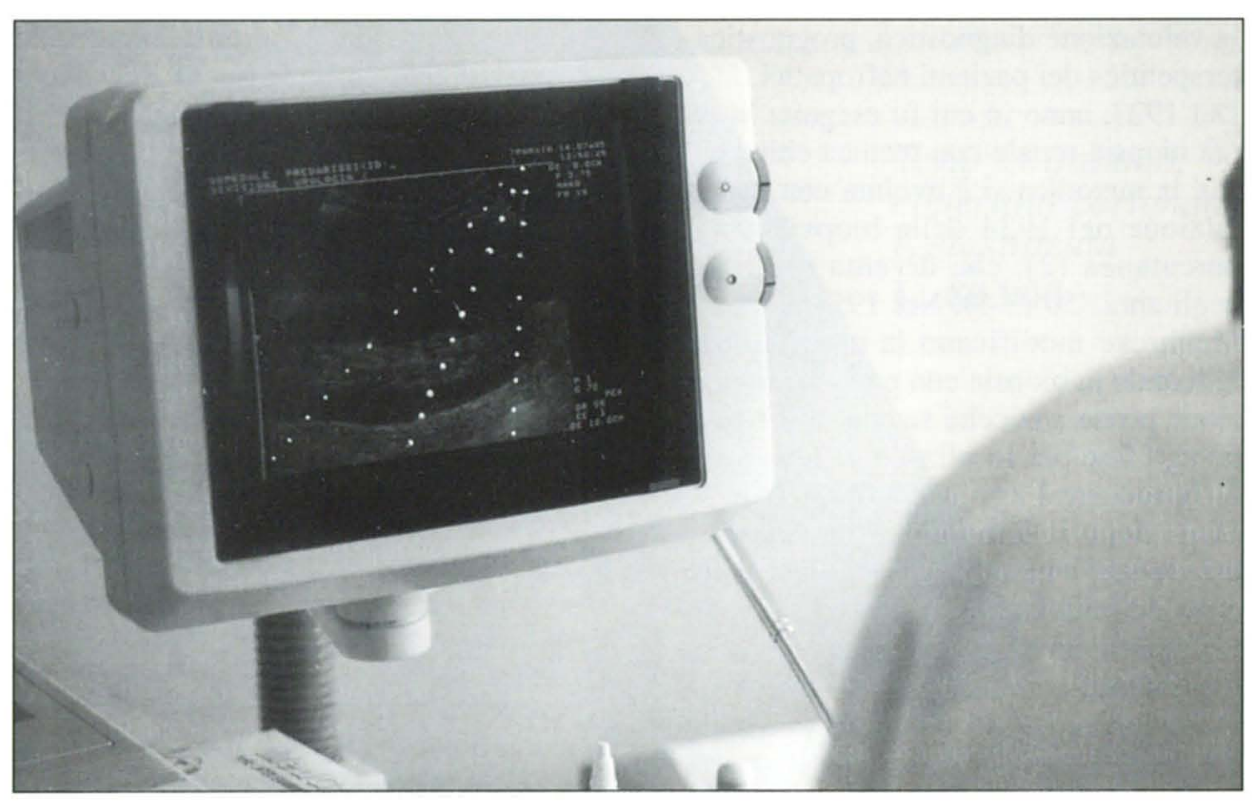

Fig. 4 - Localizzazione ecografica del polo inferiore rene sinistro e valutazione del miglior angolo di penetrazione. Sul monitor appare la traccia che seguirà l'ago nel suo percorso.

\section{Risultati}

Il materiale idoneo per la valutazione istologica, considerando tutte e tre le metodiche bioptiche, è risultato adeguato in $126 / 131$ biopsie $(96.2 \%)$. Delle 5 biopsie il cui esito aveva dato materiale inadeguato 2 erano state eseguite con tecnica transcutanea (2/26 - 7.7\%), 3 con tecnica transcutanea ecoguidata $(3 / 81-3.7 \%)$. La frequenza e la distribuzione delle complicanze delle biopsie sono riportate in Tabella I. Complessivamente si sono verificate complicanze in 39/131 (29.7\%) biopsie di cui 7/24 (29\%) nella BRC, $10 / 26(38.4 \%)$ nella BRT e 22/81 
TAB. I - PREVALENZA E DISTRIBUZIONE DELLE COMPLICANZE DELLA BIOPSIA RENALE CON TECNICA CHIRURGICA, TRANSCUTANEA E TRANSCUTANEA ECOGUIDATA

\begin{tabular}{lcccccc}
\hline Biopsie & & Sì complicanze & & & No complicanze & Totale \\
& Macroematuria & Ematoma & Anemizzazione & Altro & & \\
\hline Chirurgica & $6(25 \%)$ & - & - & $1(4.1 \%)$ & $17(70.8 \%)$ & 24 \\
Transcutanea & $6(23 \%)$ & $3(11.5 \%)$ & - & $1(3.8 \%)$ & $16(61.5 \%)$ & 26 \\
Transc. ecoguidata & $13(16 \%)$ & $8(9.8 \%)$ & $1(1.2 \%)$ & - & $59(72.8 \%)$ & 81 \\
Totale & $25(19 \%)$ & $11(8.4 \%)$ & $1(0.76 \%)$ & $2(1.5 \%)$ & $92(70.2 \%)$ & 131 \\
\hline
\end{tabular}

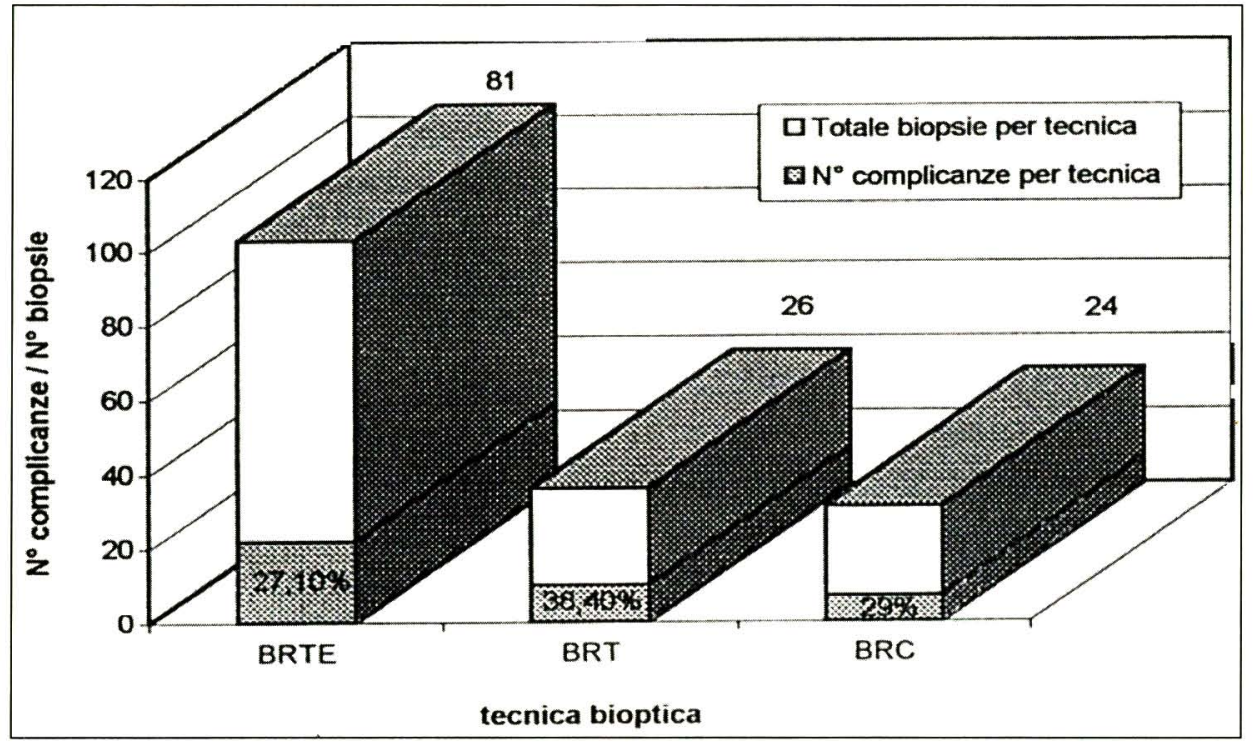

Fig. 5 - Distribuzione delle complicanze per tecnica bioptica.

(27.1\%) nella BRTE (Fig. 5). La complicanza post-bioptica più frequente è rappresentata dalla macroematuria $(25 / 131$ $19 \%$ ) di cui $6 / 24$ (25\%) nella BRC, 6/26 (23\%) nella BRT e 13/81 (16\%) nella BRTE. In 10 pazienti (40\%) la macroematuria era associata a coliche renali. L'ematoma perirenale è presente in 11/131 (8.4\%) biopsie. La distribuzione per tecnica è di $3 / 26(11.5 \%)$ per la BRT ed $8 / 81(9.8 \%)$ per la BRTE. Nell'ambito delle complicanze della biopsia renale si è verificato un singolo caso di anemizzazione di lieve entità che non ha richiesto provvedimenti terapeutici, avvenuto con la tecnica di BRTE. Tra gli eventi più significativi si segnala inoltre la puntura accidentale di parenchima epatico, uno pneumotorace e la deiscenza della ferita chirurgica avvenuti rispettivamente i primi due con la tecnica transcutanea, e il terzo con la tecnica chirurgica.

\section{Discussione}

La tecnica bioptica si è notevolmente affinata in questi ultimi anni con l'introduzione di materiali e tecnologia avanzata. L'utilizzo di aghi semiautomatici ed automatici, la possibilità di individuare ecograficamente il rene, di tracciare l'angolo di penetrazione dell'ago e di impostare lo stesso sull'adattatore per biopsia hanno condotto ad una notevole semplificazione della tecnica stessa rendendola più facile e al contempo più veloce. Con le nuove tecniche viene inoltre a ridursi la percentuale di insuccessi e la possibilità di ottenere materiale inadeguato alla analisi istologica. Nella nostra casistica, escludendo la tecnica chirurgica dove l'adeguatezza del materiale è risultata ovviamente massima, abbiamo notato una maggiore prevalenza di materiale inadeguato nella tecnica transcutanea ri- spetto alla transcutanea ecoguidata (7.7\% vs $3.7 \%)$.

La prevalenza delle complicanze della biopsia renale rimane ancora elevata sebbene, a fronte di una prevalenza globale del $29.7 \%$, si sia evidenziata nei nostri pazienti una notevole differenza tra la BRT rispetto alla BRTE, con evidente riduzione in quest'ultima (38.4\% vs $27 \%$ ) (Fig. 5). Dai risultati si evince inoltre come la prevalenza di complicanze nella BRTE sia sostanzialmente sovrapponibile alla metodica chirurgica $(27 \%$ vs $29 \%$ ). Quest'ultima è però gravata dai rischi legati alla anestesia generale, dal fatto di essere più invasiva, dal maggior stress per il paziente e l'operatore nonché dai costi di una più lunga degenza che hanno indotto gli operatori a riservare tale metodica in casi particolari. Va ricordato peraltro che le frequenze di complicanze sopra riportate sono probabilmente sottostimate rispetto alle reali in quanto non si è tenuto conto delle fistole artero-venose che possono formarsi in sede renale come conseguenza della biopsia. Ciò è dovuto al fatto che il protocollo operativo da noi adottato non prevedeva un controllo con indagine ecocolordoppler del rene biopsiato. Per quanto riguarda la natura delle complicanze, come anche riportato in letteratura, abbiamo osservato nella nostra casistica una netta prevalenza della macroematuria ( $19 \%)$ e dell'ematoma perirenale $(8.4 \%)$, sebbene quest'ultimo con una frequenza nettamente inferiore rispetto a quanto riportato da altri autori (20-22). Anche in questo caso la distribuzione per tecnica ha permesso di constatare una minore prevalenza di tali complicanze nella BRTE. È da segnalare come in $10 / 25$ pazienti $(40 \%)$ la macroematuria fosse associata a coliche renali. Tra le complicanze maggiori abbiamo osservato la 
puntura accidentale di parenchima epatico, uno pneumotorace e la deiscenza della ferita chirurgica avvenute rispettivamente le prime due con la tecnica transcutanea e la terza con la tecnica chirurgica. Come si può osservare dai nostri risultati l'introduzione della BRTE ha notevolmente ridotto l'incidenza delle complicanze maggiori della biopsia renale che in alcuni casi possono assumere i caratteri della drammaticità. Questo comporta la minimizzazione di eventi spiacevoli per il paziente legati alle complicanze stesse ed un ridotto stress per l'operatore che esegue la biopsia renale.

\section{Conclusioni}

In conclusione possiamo affermare che le complicanze di minore entità della biopsia renale sono, nonostante l'affinamento della tecnica bioptica, a tutt'oggi relativamente frequenti anche se generalmente non comportano problemi clinici di rilievo per il paziente. La metodica di BRTE riduce il rischio di complicanze della biopsia renale, offre una maggior facilità di esecuzione ed un ridotto stress per il paziente e per l'operatore. La BRTE riduce inoltre $\mathrm{i}$ tempi di esecuzione dell'esame, permette di ottenere materiale più adeguato all'analisi istologica ed evita le complicanze più gravi della biopsia renale.

\section{BIBLIOGRAFIA}

1. Gwyn NB. Biopsies and the completion of certain surgical procedures. Can Med Assoc J 1923; 13: 820-3.

2. Ball RP. Needle (aspiration) biopsy. J Tenn Med Assoc 1934; 27 : 203-6.

3. Perez-Ara A. La biopsia-punctural del rinon no megalico cosiderationes generales y aportacion de un nuevo metodo. Bol Liga Contra Cancer 1950; 25: 121.

4. Iverson P, Brun C. Aspiration biopsy of kidney. Am J Med 1951; 11: $324-30$.

5. Alwall N. Aspiration biopsy of the Kidney. Acta Med Scand 1952; 143: 430-5.

6. Kark RM, Muehrcke RC. Biopsy of kidney in prone position. Lancet 1954; 1: 1047-9.

7. Wolstenholme GEW, Cameron MP. Renal biopsy. Clinical and pathological significance. Boston, Litle, Brown, 1961.

8. Bolton WK, Tully RJ, Lewis EJ, Ranniger K. Localization of the kidney for percutaneous biopsy. An Intern Med 1974; 81: 159-64.

9. Rao K. Percutaneous renal transplant biopsy under CAT scanner guidance. Nephron 1984; 38: 273-5.

10. Birnholz JC, Kasinath BS, Corwin HL. An improved technique for ultrasound guided percutaneous renal biopsy. Kidney Int 1985; 27: 80-2.

11. Rapaccini GL, Pompili M, Caturelli E, et al. Anti M. Real-Time ultrasound guided renal biopsy in diffuse renal disease: 114 consecutive cases. Surg Endosc 1989; 3: 42-5.

12. Morrison RT, Barr JG, Wilkowski MJ, Campbell JA, Nolan CR. A new technique for percutaneous renal biopsy (Abstract). Kidney Int 1990; 37: 281 .
13. Wickre CG, Golper IA. Complications of percutaneous needle biopsy of the kidney. Am J Nephrol 1982; 2: 173-8.

14. Bach D, Hanraths M, Maar K, Grabensee B. Ultrasound guided percutaneous biopsy or operative biopsy in patients with renal impairment. Int Urol Nephrol 1988; 20: 519-23.

15. Scheehan DC, Hrapchak BB. Theory and practice of histo-technology (2nd ed.), St. Louis, Mosby, 1980.

16. Muth RG. The safety of percutaneous renal biopsy: an analysis of 500 consecutive cases. J Urol 1965; 94: 1-3.

17. Slotkin EA, Madsen PO. Complications of renal biopsy; incidence in 5.000 reported cases. J Urol 1962; 87: $13-5$.

18. River GL, Dovenbarger WV, Nikolai TF, Moffat NA. Unusual complication of kidney biopsy. J Urol 1970; 103: 15-7.

19. Parrish AE. Complications- of percutaneous renal biopsy: a review of 37 years experience. Clin Nephrol 1992; 38: 135-41.

20. Ginsburg JC, Fransman SL, Singer MA, Cohanim M, Morrin PAF. Use of computerized tomography (CT) to evaluate bleeding after renal biopsy. Nephron 1980; 26: 240-3.

21. Rosembaum R, Hoffsten PE, Stanley RJ, Klahr S. Use of computerized tomography to diagnose complications of percutaneous renal biopsy. Kidney Int 1978; 14: 87-92.

22. Alter AJ, Zimmerman S, Kirachaiwanich C. Computerized tomographic assessment of retroperitoneal hemorrhage after percutaneous renal biopsy. Arch Intern Med 1980; 140: 1323-6.

23. Meola M, Palla R, Agostini B, et al. La guida ecografica continua e gli aghi automatici semplificano la biopsia renale e riducono le complicanze post-bioptiche. Abstract del $36^{\circ}$ Congresso Nazionale SIN 1995; 12: 5. 